\title{
Brief Report: Relationship Among Viral Load Outcomes in HIV Treatment Interruption Trials
}

\section{Citation}

Treasure, Graham C., Evgenia Aga, Ronald J. Bosch, John W. Mellors, Daniel R. Kuritzkes, Michael Para, Rajesh T. Gandhi, and Jonathan Z. Li. 2016. "Brief Report: Relationship Among Viral Load Outcomes in HIV Treatment Interruption Trials." Journal of Acquired Immune Deficiency Syndromes (1999) 72 (3): 310-313. doi:10.1097/QAI.0000000000000964. http:// dx.doi.org/10.1097/QAI.0000000000000964.

\section{Published Version}

doi:10.1097/QAI.0000000000000964

\section{Permanent link}

http://nrs.harvard.edu/urn-3:HUL.InstRepos:27822404

\section{Terms of Use}

This article was downloaded from Harvard University's DASH repository, and is made available under the terms and conditions applicable to Other Posted Material, as set forth at http:// nrs.harvard.edu/urn-3:HUL.InstRepos:dash.current.terms-of-use\#LAA

\section{Share Your Story}

The Harvard community has made this article openly available.

Please share how this access benefits you. Submit a story.

Accessibility 


\title{
Relationship Among Viral Load Outcomes in HIV Treatment Interruption Trials
}

\author{
Graham C. Treasure, $*$ Evgenia Aga, MSc, $\neq$ Ronald J. Bosch, PhD, $\neq$ John W. Mellors, MD, $\S$ \\ Daniel R. Kuritzkes, MD, * Michael Para, MD,\| Rajesh T. Gandhi, MD, \ and Jonathan Z. Li, MD, MMSc*
}

\begin{abstract}
Viral load (VL) rebound timing and set point were analyzed in 235 participants undergoing analytic treatment interruption (ATI) in 6 AIDS Clinical Trials Group studies. There was no significant association between rebound timing and ATI VL set point for those who rebounded $\leq 12$ weeks. VL set points were lower in participants with rebound $>12$ weeks $(P<0.001)$ and participants treated during early infection $(P<0.001)$. Pre-antiretroviral therapy VL correlated with set point, though $68 \%$ of participants had a set point lower than pre-antiretroviral therapy VL. These results illustrate complex relationships between post-ATI virologic outcomes and the potential presence of biological factors mediating rebound timing and set point.
\end{abstract}

Key Words: HIV, treatment interruption, set point, viral rebound, outcome

(J Acquir Immune Defic Syndr 2016;72:310-313)

\section{INTRODUCTION}

Antiretroviral therapy (ART) is effective at controlling $\mathrm{HIV}$, and can suppress the plasma viral load (VL) below

Received for publication October 23, 2015; accepted February 8, 2016.

From the *Brigham and Women's Hospital, Harvard Medical School, Boston, MA; †University of North Carolina, Chapel Hill, NC; $\$$ Harvard T. H. Chan School of Public Health, Boston, MA; §University of Pittsburgh, Pittsburgh, PA; $\|$ Ohio State University, Columbus, $\mathrm{OH}$; and 9 Massachusetts General Hospital and Ragon Institute, Harvard Medical School, Boston, MA.

Supported in part by a grant from the Harvard University Center for AIDS Research (J.Z.L. and R.T.G., NIAID 5P30AI060354-08), National Institutes of Health (NIH) grants AI100699 (to Dr. Li), UM1 AI068634 (Statistical and Data Management Center of the AIDS Clinical Trials Group), UM1 AI068636 (AIDS Clinical Trials Group), and a subcontract from UM1 AI068636 to the Harvard Virology Support Laboratory (D.R.K.).

J.Z.L. has received research support from Gilead Sciences and served as a consultant for Quest Diagnostics and Merck. J.W.M. is a consultant to and has received grant support from Gilead Sciences, and is share owner of CoCrystal, Inc. D.R.K. has served as a consultant to and/or has received research grant support from Bristol-Myers Squibb, Gilead, Merck, and ViiV Healthcare; he has also received speaking honoraria from Gilead and ViiV. R.T.G. has received educational grants from Gilead, Roche and EBSCO. The other authors have no conflicts of interest to disclose.

Supplemental digital content is available for this article. Direct URL citations appear in the printed text and are provided in the HTML and PDF versions of this article on the journal's Web site (www.jaids.com).

Correspondence to: Jonathan Z. Li, MD, MMSc, Division of Infectious Diseases, Brigham and Women's Hospital, 65 Landsdowne Street, Rm 421, Cambridge, MA 02139 (e-mail: jli22@partners.org).

Copyright (c) 2016 Wolters Kluwer Health, Inc. All rights reserved. levels of detection. However, when treatment is stopped, the virus will almost inevitably rebound. The search continues for an HIV "functional cure," an intervention that allows for sustained, ART-free virologic remission. Clinical efficacy of such interventions will need to be tested in analytic treatment interruption (ATI) trials. However, the interpretation of ATI studies has been complicated by the use of varying virologic outcome measures, making comparisons and relative evaluations between ATI trials difficult.

VL set point has historically been the most commonly used virologic endpoint in ATI trials, ${ }^{1-3}$ but recent studies have also begun evaluating the timing of viral rebound in a study design that has been termed an intensely monitored antiretroviral pause. ${ }^{4-7}$ However, little is known about the relationship between these 2 virologic outcomes. In addition, it has been postulated that pre-ART VL and ATI VL set point are nearly identical ${ }^{1}$ and the difference between pre-ART VL and ATI VL set point has been used as a marker of therapeutic efficacy in previous studies. ${ }^{1,3,8-10}$ Using a pooled dataset of 6 AIDS Clinical Trials Group (ACTG) ATI studies, we performed an in-depth characterization of ATI VL endpoints.

\section{METHODS}

\section{Study Population}

Participants in 6 ACTG ATI studies (ACTG 371, ${ }^{11}$ $\mathrm{A} 5024,{ }^{12} \mathrm{~A} 5068,{ }^{13} \mathrm{~A} 5170,{ }^{14} \mathrm{~A} 5187,{ }^{15}$ and $\mathrm{A} 5197^{2}$ ) were included if they were on suppressive ART, received no recent immunologic interventions (eg, therapeutic vaccination), and had HIV-1 RNA $<50$ copies per milliliter at the time of ATI. Protocols ACTG $371^{11}$ and A5187 15 enrolled participants treated within 6 months of infection. Participants in ACTG 371 were treated for 52 weeks before the ATI; those in A5187 had to be on a stable ART with HIV-1 RNA $<50$ copies per milliliter for at least 6 months before enrollment.

\section{Statistical Analysis}

The time to viral rebound was defined as the week at which there was a confirmed HIV RNA $\geq 200$ copies per milliliter. VL set point was defined as the mean viral load between weeks 12 and 16. Associations between set point and timing of viral rebound were analyzed using Spearman correlation, Kruskal-Wallis, Mann-Whitney, and $\chi^{2}$ tests. 
The correlation of pre-ART VL vs. ATI set points was analyzed by Spearman correlation and Wilcoxon signed-rank test. We also performed a sensitivity analysis of all participants who underwent ATI in which those who discontinued the ATI before week 12 were assigned a set point viral load equal to their last known viral load.

\section{RESULTS}

\section{Participants}

Of the 235 participants, 91\% were male, 71\% were white, 14\% Hispanic, 13\% black, and 3\% others. Participants in this study had a median age of 41 years. Sixty-six percent were treated during chronic infection and 34\% during early infection. The median $\mathrm{CD}^{+}{ }^{+}$cell count at the start of ATI was 827 cells per millimeter cube. Participants treated during chronic infection had a median (Q1, Q3) of $5.1(3.2,6.5)$ years on ART.

\section{HIV Rebound Timing and Set Point}

For participants who had HIV rebound at or before week 12, there was no significant association between timing of viral rebound and the VL set point (Figs. 1A, B). However, participants who rebounded after week 12 also showed much lower set points [rebound $\leq 12(\mathrm{~N}=180)$ vs. $>12$ weeks $(\mathrm{N}=14)$ : median 4.1 vs. $1.9 \log _{10}$ HIV RNA copies per milliliter, $P<0.001]$. Lower ATI set points were more frequent among those with delayed viral rebound. Specifically, 10 of $154(6.5 \%)$ participants who rebounded within the first 4 weeks had VL set points $<1000$ RNA copies per milliliter, as compared with 7 of 57 (12.3\%) who rebounded in weeks $5-8,1$ of $10(10 \%)$ who rebounded in weeks $9-12$, and 11 of $14(79 \%)$ who rebounded after week $12\left(\chi^{2}, P<0.001 ;\right.$ Figure S1A, Supplemental Digital Content, http://links.lww.com/QAI/A796). We also performed a sensitivity analysis in which the last available VL measurement was used for 41 participants who did not reach week 12 of the ATI. The results were largely unchanged, reaffirming the original findings.

Participants treated during early infection were found to have a lower VL set point (early vs. chronic: median 3.5 vs. $4.2 \log _{10}$ HIV RNA copies per milliliter, $P<0.001$, Fig. $1 \mathrm{C}$ ). No significant difference was observed in VL set point between those on nonnucleoside reverse transcriptase inhibitor or protease inhibitor-based ART regimens.

\section{Association Between Pre-ART VL and ATI VL Endpoints}

In the 96 chronically treated participants who had both pre-ART VL and ATI set point data, there was a significant correlation between these 2 measures (Spearman $r=0.39$, $P<0.001$, Fig. 2A). The VL set point tended to be lower than the pre-ART VL with a median 2-fold decrease by patient (Q1, Q3: 5-fold decrease, 1.8-fold increase, Fig. 2B). Furthermore, $68 \%$ of participants had VL set points lower than pre-ART levels. The results were largely unchanged in
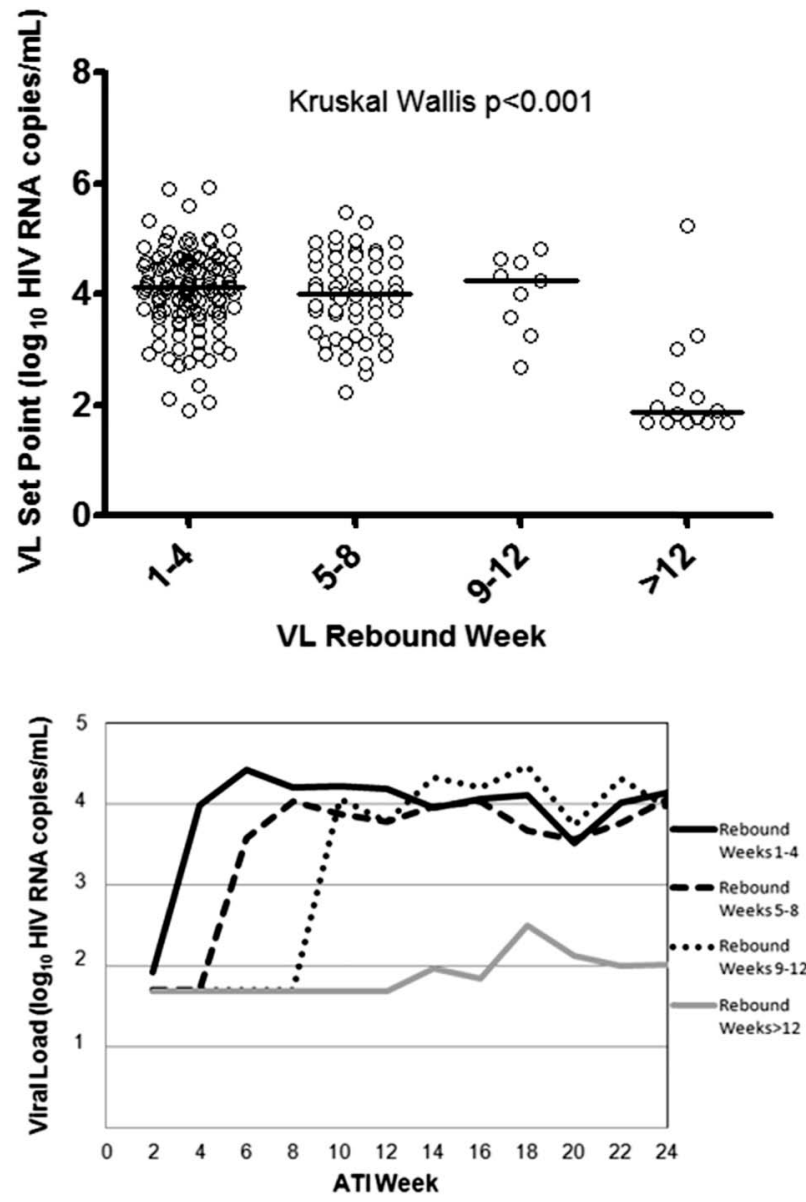

\begin{tabular}{|l|r|r|r|r|r|r|r|r|r|r|r|r|}
\hline Rebound 1-4 (N) & 100 & 154 & 88 & 125 & 65 & 100 & 40 & 87 & 40 & 23 & 23 & 58 \\
\hline Rebound 5-8 (N) & 32 & 43 & 35 & 50 & 31 & 42 & 17 & 41 & 15 & 13 & 6 & 29 \\
\hline Rebound 9-12(N) & 4 & 6 & 5 & 6 & 6 & 6 & 7 & 6 & 5 & 1 & 2 & 4 \\
\hline Rebound 13+ $(\mathrm{N})$ & 9 & 14 & 6 & 14 & 7 & 12 & 3 & 10 & 4 & 8 & 4 & 10 \\
\hline
\end{tabular}

B

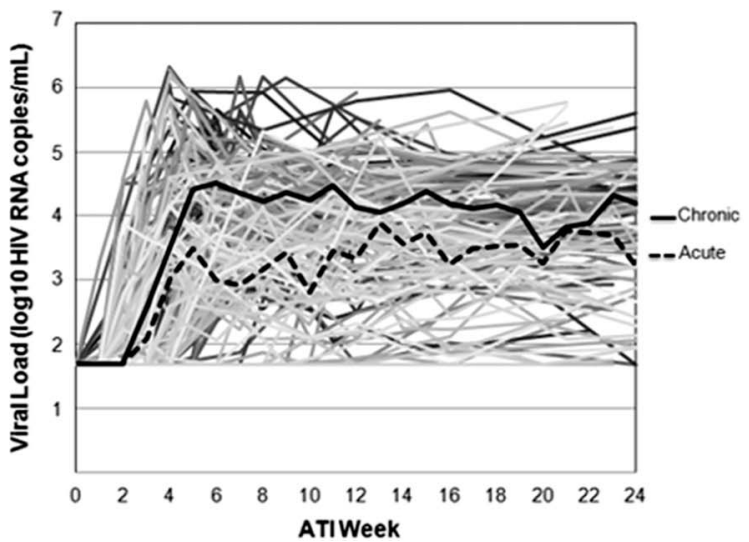

FIGURE 1. Analysis of post-ATI VL endpoints. A, VL set points and median by rebound category. B, Median VLs categorized by viral rebound week using a 2-week window for each time point with the number of observations per category per time point listed below. C, VLs for all patients shown, with median VLs of chronic and acutely treated participants in solid and dashed lines, respectively. 


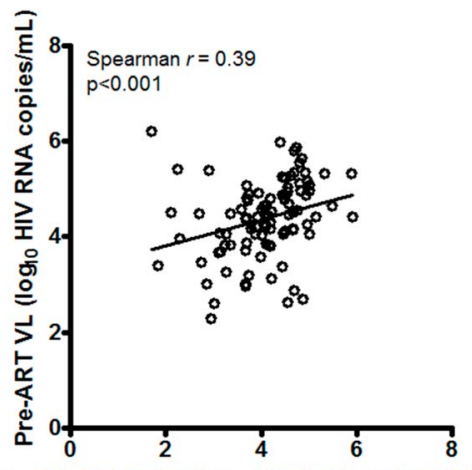

A VL Set Point $\left(\log _{10}\right.$ HIV RNA copies $\left./ \mathrm{mL}\right)$

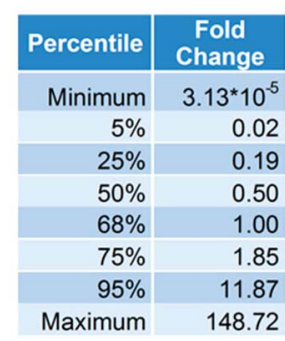

B

FIGURE 2. Relationship of pre-ART viral load and post-ATI set point. A, Plot of pre-ART VL vs. set point for 95 chronically infected patients for whom data were available. B, Percentile data for fold change of ATI VL set point to pre-ART VL (non $\log _{10}$ transformed). Fold change represents post-ATI set point divided by pre-ART viral load.

the sensitivity analysis using the last available VL for participants who did not reach ATI week 12 (pre-ART VL vs. ATI VL set point Spearman $r=0.40, P<0.001$; median 2 fold decrease; and $68 \%$ of participants with lower VL set point vs. pre-ART VL). No significant association was found between pre-ART VL and time to viral rebound, although there were relatively few participants in the viral rebound $>12$ week group with available pre-ART VL data.

\section{DISCUSSION}

HIV treatment interruption trials are expected to play a key role in evaluating the efficacy of interventions aimed at achieving sustained HIV remission. The comparison of interventions between ATI studies has been hindered by the heterogeneity in virologic outcomes and uncertainty in how to interpret trials without a placebo control arm. In this study, we found that the timing of viral rebound and ATI VL set point were largely independent when viral rebound occurred at or before ATI week 12. However, those who rebounded after week 12 were found to have significantly lower ATI VL set point. In addition, participants treated during early HIV infection were found to have lower ATI VL set point, a finding that is consistent with other studies that show favorable rebound kinetics for those treated during early stages of infection. ${ }^{16,17}$ Although we observed a correlation between pre-ART VL and ATI VL set points, the VL set point was frequently lower than the pre-ART VL with a wide range in possible outcomes.

Traditionally, ATI studies have used VL set point as a common endpoint for therapeutic efficacy. However, given the concern for prolonged participant exposure to rebound viremia, an alternative treatment interruption study design termed the intensively monitored antiretroviral pause has gained in popularity. ${ }^{4}$ This approach uses the time to viral rebound as the primary endpoint, which may entail less risk to the participants and act as a more clinically relevant outcome. However, little is known about the relationship between these

2 primary study endpoints. The results of this study show that for individuals who rebound at or before ATI week 12, there is no significant correlation between the timing of viral rebound and the VL set point. Thus, interventions that confer a modest delay in viral rebound timing may not necessarily have a significant effect on VL set point. Interestingly, we found that those who rebounded after week 12 had significantly lower VL set points, suggesting the potential presence of immunologic and/or virologic factors in this subset of participants that can both delay viral rebound and suppress the VL set point. It is important to note that even drastically delayed viral rebounds are not always associated with lower set points, especially in those whose immune system are relatively naive to HIV. This was seen in the 2 Boston patients who underwent allogeneic stem cell transplantation with both delayed viral rebound timing and high levels of VL after ATI. ${ }^{6}$

A previous study observed that the pre-ART viral load appeared to be "virtually identical" to the ATI VL set point." This observation would suggest that a change in VL between the ATI set point and pre-ART VL could be used as a study endpoint and may especially be useful in those studies without a control arm. In fact, lower ATI set point compared with pre-ART VL has been presented as evidence of therapeutic efficacy in several ATI studies. ${ }^{8-10}$ However, our results show that in more than two-thirds of participants, the ATI VL set point was lower than the pre-ART VL. The median value was a 2 -fold lower VL set point with the 25 th and fifth percentiles encompassing a 5-fold and 50-fold lower VL set points compared with pre-ART VL, respectively. There are a number of possible causes for this tendency for VL set point to be lower. These include a smaller HIV reservoir after sustained ART, ${ }^{18,19}$ immune reconstitution after ART, ${ }^{20}$ or that 16 weeks of ATI is insufficient time to allow the VL to return to pre-ART levels. ${ }^{21}$ The overall lower ATI VL set point and wide range of possible outcomes suggest that the comparison with pre-ART VL should be interpreted cautiously and may be a less accurate reflection of therapeutic efficacy than previously thought.

One notable limitation of this analysis is that a subset of individuals who initiated ATI stopped the treatment interruption phase of the studies before ATI week 12. These individuals could have potentially reached alternate criteria for ART reinitiation, including rapid viral rebound, biasing the analysis to those with lower VL set points. Therefore, we performed sensitivity analysis with a carry forward of the last recorded VLs as the VL set point for participants who did not reach ATI week 12. The results were comparable to the primary analysis, reaffirming the original conclusions. Another limitation of the analysis is that only a subset of chronically infected participants $(\mathrm{N}=96)$ have available preART VL. However, the numbers were sufficiently substantial to demonstrate the wide variance of VL set point in relation to pre-ART VL and the risks associated with the use of the comparison as an indicator of therapeutic efficacy.

HIV ATI trials represent the most conclusive strategy for evaluating the efficacy of HIV curative therapies. A detailed understanding of ATI VL endpoints is needed for the optimal design and interpretation of these trials. Our results 
revealed the complex relationship between several key virologic factors, including pre-ART VL, viral rebound timing, and VL set point. The results underscore the difficulties in interpreting uncontrolled ATI studies. In addition, the association of delayed viral rebound with suppressed VL set point suggests the presence of key immunologic and/or viral factors mediating both of these outcomes and this observation deserves further study.

\section{ACKNOWLEDGMENTS}

We thank the participants, staff, and principal investigators of the ACTG studies A371 (Paul Volberding, Elizabeth Connick), A5024 (J. Michael Kilby, Ronald Mitsuyasu), A5068 (Jeffrey Jacobson, Ian Frank, Michael Saag, Joseph Eron), A5170 (Daniel Skiest, David Margolis, Diane Havlir), A5187 (Daniel Barouch, Eric Rosenberg, Daniel Kuritzkes), and A5197 (Robert Schooley, Michael Lederman, Diane Havlir).

\section{REFERENCES}

1. Angel JB, Routy JP, Tremblay C, et al. A randomized controlled trial of HIV therapeutic vaccination using ALVAC with or without Remune. AIDS. 2011;25:731-739.

2. Schooley RT, Spritzler J, Wang H, et al. AIDS clinical trials group 5197: a placebo-controlled trial of immunization of HIV-1-infected persons with a replication-deficient adenovirus type 5 vaccine expressing the HIV-1 core protein. $J$ Infect Dis. 2010;202:705-716.

3. Garcia F, Climent N, Guardo AC, et al. A dendritic cell-based vaccine elicits T cell responses associated with control of HIV-1 replication. $\mathrm{Sci}$ Transl Med. 2013;5:166ra2.

4. Li JZ, Smith DM, Mellors JW. The need for treatment interruption studies and biomarker identification in the search for an HIV cure. AIDS. 2015;29:1429-1432.

5. Number of persons tested for HIV—United States, 2002. MMWR Morb Mortal Wkly Rep. 2004;53:1110-1113.

6. Henrich TJ, Hanhauser E, Marty FM, et al. Antiretroviral-free HIV-1 remission and viral rebound after allogeneic stem cell transplantation: report of 2 cases. Ann Intern Med. 2014;161:319-327.

7. Rothenberger MK, Keele BF, Wietgrefe SW, et al. Large number of rebounding/founder HIV variants emerge from multifocal infection in lymphatic tissues after treatment interruption. Proc Natl Acad Sci U S A. 2015;112:E1126-E1134

8. Pollard RB, Rockstroh JK, Pantaleo G, et al. Safety and efficacy of the peptide-based therapeutic vaccine for HIV-1, Vacc-4x: a phase 2 randomised, double-blind, placebo-controlled trial. Lancet Infect Dis. 2014;14:291-300

9. Gandhi RT, O'Neill D, Bosch RJ, et al. A randomized therapeutic vaccine trial of canarypox-HIV-pulsed dendritic cells vs. canarypox-HIV alone in HIV-1-infected patients on antiretroviral therapy. Vaccine. 2009; 27:6088-6094.

10. Tebas P, Stein D, Tang WW, et al. Gene editing of CCR5 in autologous CD4 T cells of persons infected with HIV. N Engl J Med. 2014;370:901-910.

11. Volberding P, Demeter L, Bosch RJ, et al. Antiretroviral therapy in acute and recent HIV infection: a prospective multicenter stratified trial of intentionally interrupted treatment. AIDS. 2009;23:1987-1995.

12. Kilby JM, Bucy RP, Mildvan D, et al. A randomized, partially blinded phase 2 trial of antiretroviral therapy, HIV-specific immunizations, and interleukin-2 cycles to promote efficient control of viral replication (ACTG A5024). J Infect Dis. 2006;194:1672-1676.

13. Jacobson JM, Pat Bucy R, Spritzler J, et al. Evidence that intermittent structured treatment interruption, but not immunization with ALVACHIV vCP1452, promotes host control of HIV replication: the results of AIDS Clinical Trials Group 5068. J Infect Dis. 2006;194:623-632.

14. Skiest DJ, Su Z, Havlir DV, et al. Interruption of antiretroviral treatment in HIV-infected patients with preserved immune function is associated with a low rate of clinical progression: a prospective study by AIDS Clinical Trials Group 5170. J Infect Dis. 2007;195:1426-1436.

15. Rosenberg ES, Graham BS, Chan ES, et al. Safety and immunogenicity of therapeutic DNA vaccination in individuals treated with antiretroviral therapy during acute/early HIV-1 infection. PLoS One. 2010;5:e10555.

16. Hamlyn E, Ewings FM, Porter K, et al. Plasma HIV viral rebound following protocol-indicated cessation of ART commenced in primary and chronic HIV infection. PLoS One. 2012;7:e43754.

17. Steingrover R, Pogany K, Fernandez Garcia E, et al. HIV-1 viral rebound dynamics after a single treatment interruption depends on time of initiation of highly active antiretroviral therapy. AIDS. 2008;22:1583-1588.

18. Besson GJ, Lalama CM, Bosch RJ, et al. HIV-1 DNA decay dynamics in blood during more than a decade of suppressive antiretroviral therapy. Clin Infect Dis. 2014;59:1312-1321.

19. Laanani M, Ghosn J, Essat A, et al. Impact of the timing of initiation of antiretroviral therapy during primary HIV-1 infection on the decay of cell-associated HIV-DNA. Clin Infect Dis. 2015;60:1715-1721.

20. Al-Harthi L, Siegel J, Spritzler J, et al. Maximum suppression of HIV replication leads to the restoration of HIV-specific responses in early HIV disease. AIDS. 2000;14:761-770.

21. Fauci AS, Pantaleo G, Stanley S, et al. Immunopathogenic mechanisms of HIV infection. Ann Intern Med. 1996;124:654-663. 